\title{
Distributed Control of Multiple Electric Springs for Voltage Control in Microgrids
}

\author{
X. Chen, Member, IEEE, Y. Hou, Member, IEEE, and S.Y.R. Hui, Fellow, IEEE
}

\begin{abstract}
Dispersed distribution of many inverter-based Electric springs (ESs) over the power grid as a means to provide stability support for smart grid against high penetration of intermittent renewable power has been suggested recently. While single ES has its own local controller, their wide dispersion makes it difficult to coordinate their functions. In this paper, a novel distributed control method for adjusting the whole voltage level of ESs installed in the network is developed. Firstly, the specific control scheme for the single ES is designed to ensure good transient response and zero steady-state error. Combining with the consensus algorithm for ES reference voltage updating, the control strategy for multiple ESs is implemented, in which the inductor current is considered as the feedback variable to modify the output of the voltage controller. Compared with the conventional droop control, the scheme can maintain the voltage level of the critical load without sacrificing the voltage control accuracy. In addition, this algorithm guarantees system stability and optimal convergence speed. Simulation results show that the distributed voltage control can guarantee the overall coordination of parallel ESs under various operation conditions.
\end{abstract}

Index Terms-Electric springs, distributed control, microgrid, renewables.

\section{INTRODUCTION}

In a distribution network, variability and uncertainty associated with renewable energy sources, such as wind power and solar power, can easily incur the power fluctuation, voltage and frequency instability[1]-[3]. ESs are proposed and intended to support the voltage at the connection point across the distribution network [4].

This work was supported by the HK Research Grant Council under the Collaborative Research Fund (HKU10/CRF/10), National Natural Science Foundation of China (51277155), The University of Hong Kong (Seed Projects: 201111159239 \& 201203159010; Theme-based Research Scheme: T23-701/14-R/N), National Basic Research Program of China (973 Program:

2012CB215102), and HK Research Grant Count under Early Career Scheme (HKU 739713E)

X. Chen, and Y. Hou are with the Department of Electrical \& Electronic Engineering, The University of Hong Kong, Pokfulam Road, Hong Kong, China (e-mail: chenxia@eee.hku.hk; yhhou@eee.hku.hk).

S. Y. Hui is with the Departments of Electrical \& Electronic Engineering, University of Hong Kong and also with Imperial College London, SW7 2AZ, U.K. (e-mail: ronhui@eee.hku.hk; r.hui@imperial.ac.uk).
The application of the inverter based ESs is a innovative solution for distributed voltage control through effective demand-side management. In order to ensure the voltage constant at the parallel critical load (C), ES is connected in series with the uncritical load (NC) that are less sensitive to the voltage fluctuation. Previous studies about ESs mainly focused on the single ES. In [5] and [6], the dynamic modeling and steady-state analysis of ES are given to prove the capability of ES in providing different types of power/voltage compensations to the load. A comparison between the ES and Static synchronous compensator (STATCOM) shows that a group of ESs achieves better total voltage regulation than STATCOM with less overall reactive power capacity [7]. The effect of ESs in reducing energy storage requirements is theoretically proven and practically demonstrated [8]. The mitigation of the frequency and voltage fluctuation in microgrid using ES is verified in [9]. Three-phase ES circuit and its operation in reducing the power imbalance are described in [10]. [11] presents a droop control method for parallel operation of distributed ESs for stabilizing ac voltage. However, the voltage reference for ES control needs to be improved accurately.

If ESs are massively installed in the network, they can provide highly distributed and robust support for the smart grid, similar to the arrays of mechanical springs supporting the mattress. Since low voltage microgrid is featured as the resistive line impedance and a high share of intermittent renewables, when the penetration of power electronically interfaced ESs installed in the network increases, it may cause the system susceptible to the oscillation in the case of network disturbances. In addition, due to the power variation of the renewable energy as well as the line impedance mismatch between the ESs, the reactive power unbalance will occur in the network and thus the voltage reference can't be set to the same value to avoid the unstable operation of ESs as illustration in [11]. Besides, the "plug and play" feature of ES poses a substantial complexity on the control of multiple ESs. It requires the installation of additional ESs should not change the existing control of ESs in the microgrid. Consequently, it is necessary to develop a general control scheme to derive the specific voltage reference for ES at different installation points and improve the voltage and reactive power control accuracy.

The conventional centralized control based on the sophisticated communication network made it difficult to update the data and share the dynamic signal when ESs are scattering all over the network [12][13]. In addition, it is expensive to col- 
lect the globe information, and it is susceptible to the single point failure when handling huge amount of data. Besides, the method is lack of adaptability to reach the operation requirement of the plug-and-play of ESs [14]. An alternative one is the droop control method. It avoids the intercommunication and has the independent and autonomous features. The droop control is utilized widely by emulating the droop characteristics of synchronous generators to adjust the output voltage frequency and magnitude of the connection point voltage[15][17]. However, it has some potential drawbacks which have been widely discussed. For example, the drawbacks of the droop control lie in the slow transient response, high dependency on the inverter output impedance and the power sharing inaccuracy. In contrast to the frequency, the voltage is not a global variable and the reactive power control will incur the adverse impact on the voltage regulation for the critical load

To overcome these limitations, it is necessary to develop a control strategy to crack the problems associated with the existing methods. The control strategy should improve the control accuracy and robustness, meanwhile minimize the communication cost. The consensus control enables different agents in the same group to work toward the same objective by only using the neighboring agent information [20].

The paper highlights a novel fully distributed control concept proposed for the coordination control of multiple ESs to overcome the disadvantages brought by the conventional control. To calculate the desired voltage level, the discrete consensus algorithm is proposed, which can guarantee the convergence and can be implemented by simple communication network. Each ES receives the updating voltage reference signal and realizes its network synchronization according to the discovered information. The voltage reference will be propagated among the ESs through the communication topology designed. The rest of this paper is outlined as follows. Section II discusses the single ES control, and the distributed control for multiple ESs are presented in Section III. The consensus control is introduced in Section IV. The controller performance is verified by simulation in Section V. Section VII concludes the paper.

\section{SINGLE ES CONTROL}

The problem to be solved is how to coordinate the power electronic devices installed in the grid and realize the cooperative control of ESs in a distributed manner. In Fig.1, in a microgrid, each ES is assigned with an agent and each agent knows local information through the communication flow spanning among ESs to facilitate data exchange. The communication infrastructure is independent of the physical structure of the location of ESs. This kind of distributed control mode can realize the plug-and-play of ESs. The failure of one ES will not make the system susceptible to voltage problems.

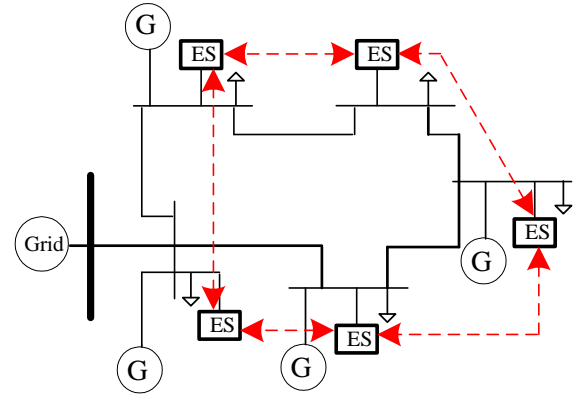

Fig. 1 Microgrid with DG and ES in parallel to a common ac bus

As all ESs installed in the network are independent of each other, the study of multiple ESs begins with the specific controller design for the single ES. The ESs can be connected to the network by the bypass switches and can always keep online if necessary. If the ES encounters any problem, it can be switched off and the non-critical load will not be affected. The ES consists of an inverter with the battery installed on the dc link and a LC filter on the ac side. The output of ES is in series with the uncritical loads such as air conditioner, heater and refrigerator, which can tolerate small range of voltage variation. However, there still exists a minimum voltage for the non-critical loads so that these loads can still operate within the specifications. The voltage tolerance of the noncritical load mainly depends on the load type. For example, some lighting systems have an operating input phase voltage in the range of $170 \mathrm{~V}-265 \mathrm{~V}$ for the markets using $230 \mathrm{~V}$ as nominal mains voltage [10]. The combination of ES and uncritical load forms the smart load to ensure the voltage regulation of the parallel critical load. The smart load can be intended to provide reactive power compensation to the system by adjusting the output voltage and suppress the voltage fluctuation at the critical load. It can realize an accurate voltage regulation with prompt dynamic response.

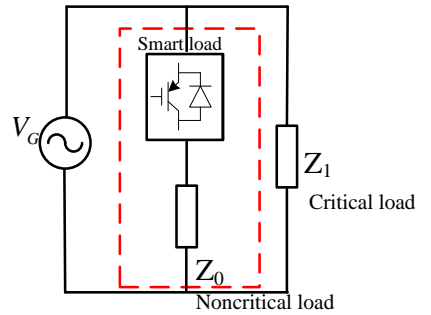

Fig.2 Configuration of the smart load

In Fig.2, the ES provides a voltage series with the noncritical load $Z_{o}$ to regulate the voltage of the parallel critical load $Z_{1}, V_{G}$ is the terminal voltage of the renewable. The proportion of the two kinds of loads poses a significant impact on the voltage regulation capability and the reactive power output of ES. The voltage regulation capability of an ES is dependent on the relative proportion of $\mathrm{NC}$ and $\mathrm{C}$ load. A higher proportion of noncritical load indicates a high voltage regulation capability of ES. The per-phase capacitance and inductance of the filter are denoted by $C$ and $L$, respectively. The RL type of load is chosen to be the allocation place for ES. $Z_{o}$ is the impedance of the inductance type of load. $Z_{1}$ is the impedance of the critical load. $Z_{2}$ is the 
equivalent line impedance. $V_{s}, V_{\mathrm{es}}$ and $V_{a c}$ are the voltage of the critical load, the ES voltage, the output voltage of the inverter. $I_{1}, I_{2}$ and $I_{3}$ are the line current, the critical load current, the non-critical load current. $I_{L}$ and $I_{c}$ are the inductance and capacitor current. The equivalent circuit for ES is shown in Fig.3 (a).
Fig.3 (a) ES simplified circuit

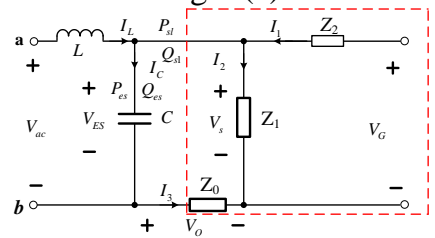

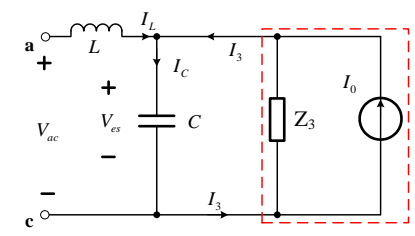

Fig.3 (b) Equivalent circuit
In Fig.3(b), the equivalent impedance $Z_{3}$ and the equivalent current $I_{0}$ can be written as

$$
\begin{aligned}
& Z_{3}=\frac{Z_{1} Z_{2}+Z_{1} Z_{0}+Z_{0} Z_{2}}{Z_{1}+Z_{2}} \\
& I_{0}=V_{G} \times \frac{Z_{1}}{Z_{1} Z_{2}+Z_{1} Z_{0}+Z_{0} Z_{2}}
\end{aligned}
$$

In Fig.3(a):

$$
s C V_{e s}(s)=I_{L}(s)+\frac{V_{S}(s)-V_{e s}(s)}{Z_{3}}
$$

In Fig.3(b):

$$
\begin{gathered}
s L I_{L}(s)=V_{a c}(s)-V_{e s}(s) \\
s C V_{e s}(s)=I_{L}(s)+I_{0}(s)-\frac{V_{e s}(s)}{Z_{3}}
\end{gathered}
$$

The relationship between the critical load voltage $V_{s}$ and the output voltage of the inverter $V_{a c}$ can be obtained

where

$$
\frac{V_{s}}{V_{a c}}=G_{v}
$$

$$
G_{v}(s)=\frac{Z_{1} Z_{2}}{\left(Z_{1}+Z_{2}\right)\left(Z_{3} L C s^{2}+L s+Z_{3}\right)}
$$

The inductor current $I_{L}$ has the following relationship with $V_{s}$ and $V_{a c}$,

$$
\begin{gathered}
I_{L}=\frac{V_{a c}\left(1+s C Z_{0}\right)-V_{s}}{s^{2} L Z_{0}+s L C+Z_{0}} \\
G_{2}(s) V_{a c}=I_{L}+G_{3}(s) V_{s}
\end{gathered}
$$

in which

$$
\begin{aligned}
& G_{2}(s)=\frac{1+s C Z_{0}}{s^{2} L Z_{0}+s L C+Z_{0}} \\
& G_{3}(s)=\frac{1}{s^{2} L Z_{0}+s L C+Z_{0}}
\end{aligned}
$$

The active power $P_{e s}$ and the reactive power $Q_{e s}$ from the ES are given by

$$
\begin{aligned}
P_{e s}=\frac{\left|V_{a c}\right| \cdot\left|V_{e s}\right| \cdot \sin \left(\theta_{a c-e s}\right)}{X_{L}} \\
Q_{e s}=\frac{\left|V_{e s}\right|^{2}-\left|V_{a c}\right| \cdot\left|V_{e s}\right| \cdot \cos \left(\theta_{a c-e s}\right)}{X_{L}}
\end{aligned}
$$

(9)

in which $\theta_{a c-e s}$ is the phase difference between the voltage denoted.

The active power $P_{s l}$ and the reactive power $Q_{s l}$ provided by the smart load to the system are derived as

$$
\begin{aligned}
P_{s l} & =\frac{\left|V_{a c}\right| \cdot\left|V_{e s}\right| \cdot \sin \left(\theta_{a c-e s}\right)}{X_{L}}+\frac{\left(\left|V_{s}\right|-\left|V_{e s}\right|\right)^{2} \cdot \sin \left(\theta_{L}\right)}{Z_{0}} \\
Q_{s l} & =\frac{\left|V_{e s}\right|^{2}-\left|V_{a c}\right| \cdot\left|V_{e s}\right| \cdot \cos \left(\theta_{a c-e s}\right)}{X_{L}}+\frac{\left(\left|V_{s}\right|-\left|V_{e s}\right|\right)^{2} \cdot \cos \left(\theta_{L}\right)}{Z_{0}}
\end{aligned}
$$

It can be seen that the voltage magnitude and the phase of ES impact the power flows supplied by the inverter to the load.

\section{CONTROLLER FOR MULTIPLE ESS}

A distributed control approach is proposed to mitigate the voltage problem caused by the variable output power of renewable. The conventional droop controller emulates the actual P-f and Q-V droop relations of a synchronous generator, which can offer the inertia response to the system. In microgrid, the control method is utilized for multiple inverters power sharing. The controller adjusts the reference voltage magnitude and frequency for the voltage control loop as follows

$$
\begin{aligned}
& \omega=\omega^{*}-m_{P}\left(P-P^{*}\right) \\
& V=V^{*}-n_{q}\left(Q-Q^{*}\right)
\end{aligned}
$$

where $m_{p}$ and $n_{q}$ are the frequency and the voltage droop coefficient, respectively. $\omega^{*}$ and $V^{*}$ are the frequency and voltage references. $P^{*}$ and $Q^{*}$ are the active power and reactive power references.

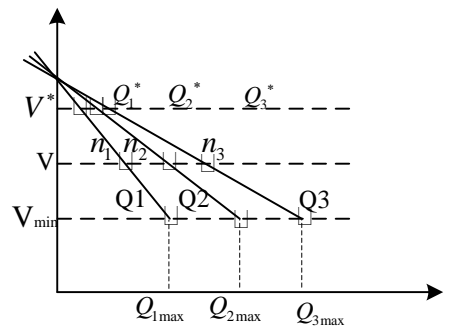

Fig.4 Q-V droop control

The conventional droop control implemented according to Equ.(10) and Equ.(11) often impacts the power sharing accuracy brought by its dependency on the line impedances [18].

The parallel ESs control introduces the inductor current to produce the voltage droop $V_{L}$ to modify the control signal $V_{v c}$. $V_{p c}$ is the final input signal for PWM modulator. $V_{a c}$ is the output ac voltage of the inverter. Each ES employs its own inductor current to ensure its proper parallel operation as shown in Fig.5.

The resonant controller is intended to replace the integrator to achieve a high gain and ensure zero steady-state error regulation. The transfer function of proportional resonant (PR) controller is 


$$
G_{1}(s)=k_{p}+k_{r} \frac{2 \omega_{c} s}{s^{2}+2 \omega_{c} s+\omega_{0}^{2}}
$$

where $k_{p}$ and $k_{r}$ are the proportional gain and the resonant gain, respectively. $\omega_{0}$ is the resonant frequency and $\omega_{0}=100 \pi \mathrm{rad} / \mathrm{s} \cdot \omega_{c}$ is the cutoff frequency for the resonant main bandwidth control.

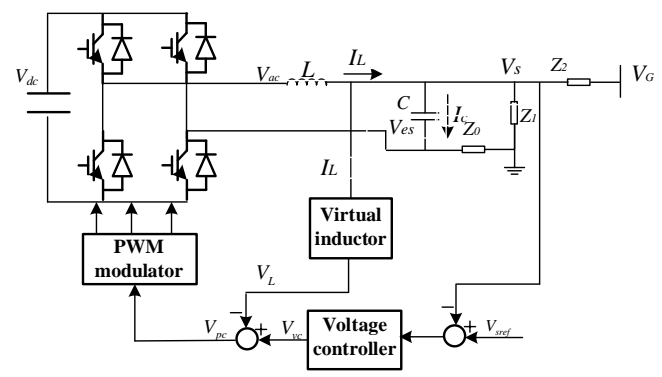

Fig.5 The schematic of the control system

It is shown that the controller has a wide bandwidth around the resonant frequency. The controller sensitivity to slight frequency variation is reduced if the resonant peak decreases. The transient performance of the ES with PR controller mainly depends on the proportional gain $k_{p}$. With a larger $k_{p}$, the system bandwidth is broaden and the response to a change in the reference signal accelerates. On the other hand, it will cause transient resonant oscillations or cause the stability problem since the LC resonance is amplified. The resonant gain $k_{r}$ determines the peak value, and a larger $k_{r}$ will not affect the transient and stability of the system. $k_{p}=0.2$ $k_{r}=1000$ are finally assigned to the controller as the method mentioned in [19]. The cutoff frequency determines the resonant bandwidth of the controller. The increasing cutoff frequency $\omega_{c}$ may yield a less sensitive voltage controller. The practical arrange for the cutoff frequency ranges from 10 to $100 \mathrm{rad} / \mathrm{s}$ for satisfactory results.

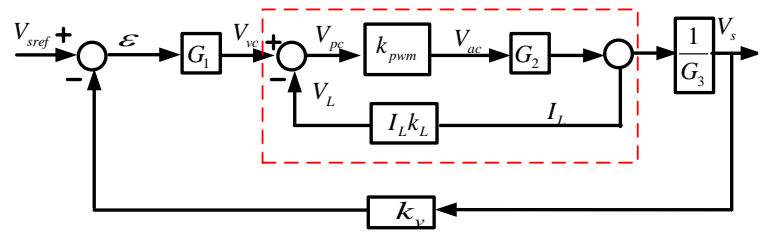

Fig.6 The schematic of the control system

In Fig.6, the double-loop control is introduced for the ESs control. The outer-loop voltage controller is mainly utilized for the ES voltage control at the connection point. The innerloop controller adopts the inductor current as the feedback variable to modify the output of the voltage controller. Hence, the signal posed on the pulse width modulation (PWM) modulator is changed.

The control strategy for multiple ESs are implemented specifically as the scheme shown below

$$
\left\{\begin{array}{l}
V_{p c}=V_{v c}-V_{L} \\
V_{L}=I_{L} k_{L}
\end{array}\right.
$$

where $k_{L}$ is the droop gain selected.

The voltage control signal from the outer-loop control subtracts the voltage drop caused by the filter current, that is, the inverter voltage is reduced according to the increasing inductor current.

The relationship between the signal sent to the PWM modulator and the output voltage of the inverter is described as

$$
V_{a c}=k_{p w m} V_{p c}
$$

where $V_{p c}$ is the input voltage for the inverter, $k_{p w m}$ is the modulating gain.

The gain of the PWM modulator can be calculated below

$$
k_{p w m}=\frac{V_{d c}}{V_{t r i}}
$$

in which, $V_{d c}$ and $V_{t r i}$ denote the dc-link voltage and the PWM carrier voltage, respectively.

$$
V_{a c}=k_{p w m}\left(V_{v c}-I_{L} k_{L}\right)
$$

The equation shows that the current variation in $I_{L}$ leads to the voltage $V_{a c}$ fluctuation. Combining with the current $I_{L}$, $k_{L}$ may be considered as the virtual resistance causing the voltage droop for multiple parallel ESs in the network.

$$
\begin{gathered}
V_{a c}=s L I_{L}+V_{e s}=k_{p w m}\left(V_{v c}-I_{L} k_{L}\right) \\
V_{e s}=k_{p w m} V_{v c}-I_{L}\left(k_{p w m} k_{L}+s L\right)
\end{gathered}
$$

The ES voltage can be rewritten as

$$
V_{e s}=k_{p w m} V_{v c}-I_{L} Z^{*}
$$

If the gain is selected to be larger than the induction, the inductance effect can be ignored and the output impedance presents the purely resistive characteristics. The inverter is deemed as a voltage source cascaded with a resistive output impedance. This strategy proposed has the basic principles of droop control, because it decreases the inverter output voltage $V_{a c}$ with increasing inductor current $I_{L}$.

The phase locked loop (PLL) is employed to obtain the phase angle and the voltage magnitude is given strictly according to the voltage requirement for the critical load. The voltage reference $V_{\text {sref }}$ can be achieved by the control algorithm proposed below.

By contrast, the scheme utilizes the instantaneous values to provide a dynamic response suitable for the multiple inverters instead of modifying the voltage reference $V_{\text {sref }}$ directly. The control principle is similar to the droop control, which decreases the input voltage of the LC filter with the increasing inductor current. The voltage of the critical load can be kept constant at the reference value. The output impedance of the closed-loop inverter affects the power-sharing accuracy and also determines the droop control strategy. The scheme can maintain the voltage constant without sacrificing the voltage level of the critical load as the conventional droop 
control does.

\section{CONSENSUS CONTROL}

The consensus control is introduced to obtain the reference voltage assigned to ES. Each ES can access the local voltage and load information, but can't acquire the global information. The average consensus theory is intended to ensure the information exchange in a distributed mode based on the local information of agents [20]-[24]. The agents update the value of their information states based on the information state of their neighbors[25]. The goal is to design an update law so that the information states of all agents converge to a common value. The consensus algorithm is conceived for negotiating to reach consensus and provide a systematic consensus mechanism to computer the consensus value. An iterative form of the consensus algorithm can be stated in discrete-time as:

$$
x_{i}^{k+1}=x_{i}^{k}+\sum_{j=1}^{n} a_{i j}\left(x_{j}^{k}-x_{i}^{k}\right)
$$

where $\mathrm{n}$ is the number of agents in the microgrid. $a_{i j}$ is the communication weight for data transfer from node $i$ to node $j$. If nodes are not connected through a communication line, $a_{i j}=0$. The information state $x_{i}^{k}$ is driven toward the information states of its neighbors $x_{j}^{k}$ according to the weighted average of its current state and the current states of its neighbor. The agent maintains its current information state if it does not exchange information with other agent at that instant.

In this case, the overall information discovery process is modelled as a discrete time linear system and the flow chart of the algorithm is shown in Fig.7.

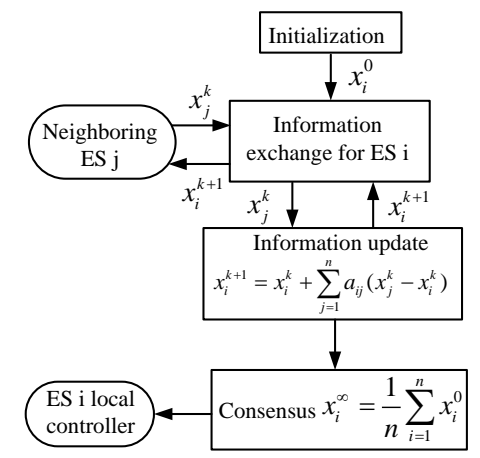

Fig.7 Flow chart for the consensus control

The global information discovery process of the whole microgrid can be represented using matrix format as follows

$$
\mathbf{X}^{\mathbf{k}+1}=\mathbf{D} \mathbf{X}^{\mathbf{k}}
$$

where $\mathbf{D}$ is the weight matrix of the communication network. $\mathbf{X}^{\mathbf{k}}$ and $\mathbf{X}^{\mathbf{k}+1}$ are the vectors of discovered information at the $\mathrm{k}^{\text {th }} \quad$ and $(\mathrm{k}+1)^{\text {th }} \quad$ iterations respectively. $\mathbf{X}^{\mathbf{k}}=\left[x_{1}^{k}, \ldots, x_{i}^{k}, \ldots, x_{n}^{k}\right]$

If the constant edge weight $\varepsilon$ is considered, $\mathbf{D}$ can be described as $\mathbf{D}=\mathbf{I}-\varepsilon \mathbf{L}$ in Equ.(20) . $\mathbf{L}$ is the Laplacian matrix of the communication network. Mathematically, the communication system for the agents can be described by a communication matrix $\mathbf{D}$, i.e., $a_{i j}=a_{j i}$. $\mathbf{D}$ can be designed to be symmetrical, and is called the doubly stochastic matrix and all eigenvalues of $\mathrm{D}$ are less than or equal to 1 .

$$
\mathbf{D}=\left(\begin{array}{ccc}
1-\sum_{j \in N_{1}} a_{1 j} & \cdots & a_{1 n} \\
\vdots & \ddots & \vdots \\
a_{n 1} & \cdots & 1-\sum_{j \in N_{n}} a_{n j}
\end{array}\right)
$$

The matrix $\mathbf{D}$ is designed to be symmetrical $a_{i j}=a_{j i}$ and satisfy the following conditions as:

(1) the sums of rows and columns are all one;

(2) the eigenvalue $\left|\lambda_{I}\right|<1$, for $i=2, \ldots, n$

For the stability analysis, only $a_{i j}$ satisfying the following condition, the stability of the designed algorithm can be guaranteed.

$$
0<a_{i j}<\frac{2}{n_{i}+n_{j}}
$$

where $n_{i}$ and $n_{j}$ are the numbers of agents in the neighborhood of agent $i$ and agent $j$, respectively.

The final consensus equilibrium $\mathbf{X}_{\mathrm{eq}}$ :

$$
\mathbf{X}_{\mathrm{eq}}=\lim _{k \rightarrow \infty} \mathbf{X}(\mathrm{k})=\lim _{k \rightarrow \infty} \mathbf{D}^{k} \mathbf{X}(0)=\frac{1}{n} \mathbf{1} \cdot \mathbf{1}^{T}
$$

where $\mathbf{X}(0)$ is the vector of the initial values hold by each agent. 1 denotes the vector with all the elements 1 . The detail proof of the convergence can be found in [27]

By initializing $x_{i}$ with the locally measured voltage, the algorithm can search the averages of the required global information. Then the desired voltage level can be calculated and updated constantly according to the variable renewables.

To adapt to the network topology change caused by the plug-and-play of ESs, the adaptive weights updating rule is illustrated as

$$
a_{i j}=\left\{\begin{array}{lr}
\frac{1}{\left[\max \left(n_{i}, n_{j}\right)+1\right]} & j \in N_{i} \\
1-\sum_{j \in N_{i} \frac{1}{\left[\max \left(n_{i}, n_{j}\right)+1\right]}} & i=j \\
0 & \text { otherwise }
\end{array}\right.
$$

By applying this method, an agent can adjust its coefficients locally according to the topology change.

A two-level control strategy is deployed in the microgrid. The upper level control discovers the voltage reference for the ES through the consensus algorithm. It measures and initializes the local information. The local information update module is responsible for updating the ES output voltage reference according to equ. (19). The information exchange module exchanges the information with its neighboring ESs. A simple supporting communication topology is designed to be identical to the physical network based on cost, location, 
convenience, etc. The lower level control implements the reference voltage tracking of ES as the control strategy designed in Fig.5.

\section{SIMULATION STUDIES}

To test the effectiveness of the proposed approach, the models of a nine-bus self-governing microgrid with three ESs are established in PSCAD/EMTDC. The agents model with communication links and the consensus algorithm are designed in Matlab with 100ms time step. As shown in Fig.8, User-defined interface (UDI) models in PSCAD can realize interaction among the agents and the microgrid. The data in PSCAD, such as the agent index, number of neighbors, and the voltage can be collected in MATLAB. The agents modeled in MATLAB can then exchange data with their neighbors and discover the global information using the consensus algorithm. Final, the voltage control reference values will be sent back to PSCAD by the interface to implement the distributed control of each ES locally. The communication time delay is not considered here.

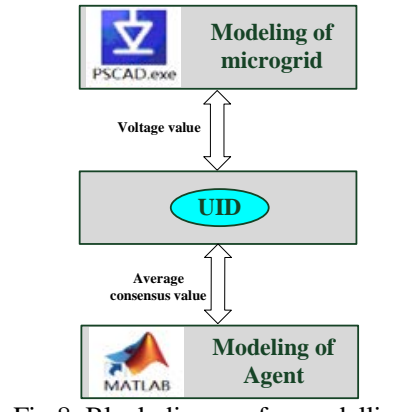

Fig.8 Block diagram for modelling

Fig.9 illustrates the test system topology. The microgrid consists of three DGs, in which G1 is replaced with the double-fed induction generator (DFIG) based wind turbine. The voltage level for the system is $35 \mathrm{kV}$ and the rated power for the G1, G2 and G3 are 8 MVA, 6 MVA and 4 MVA, respectively. The parameters for the generators and the network can be referred to [9].The three ESs are installed at bus 8 , bus 6 and bus 5 , respectively. The communication topology is designed to be the same as the topology of the system configuration.

\begin{tabular}{|c|c|c|c|c|c|}
\hline Para. & $k_{p}$ & $k_{r}$ & $k_{v}$ & $k_{p w m}$ & $k_{L}$ \\
\hline ES & 0.26 & 160 & 0.02 & 0.85 & 0.016 \\
\hline
\end{tabular}

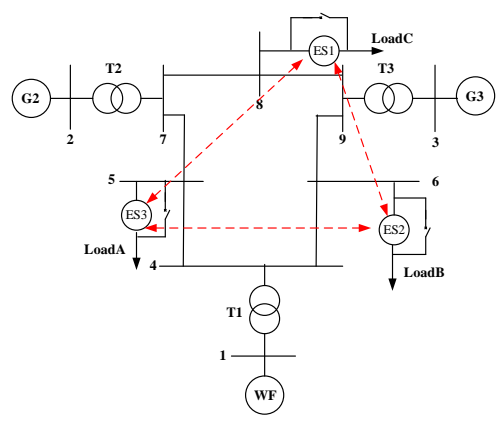

Fig. 9. Nine-bus system
The Laplacian matrix of the communication network is designed as:

$$
\mathbf{L}=\left(\begin{array}{ccc}
2 & -1 & -1 \\
-1 & 2 & -1 \\
-1 & -1 & 2
\end{array}\right)
$$

The constant edge weight $\varepsilon$ impacts the dynamic of the discrete algorithm. The eigenvalues of $\mathbf{L}$ are $\left[\begin{array}{lll}0 & 3 & 3\end{array}\right]^{T}$. The optimal $\varepsilon=1 / 3$ is given to achieve fast convergence speed. The corresponding D matrix is obtained:

$$
D=\left(\begin{array}{lll}
\frac{1}{3} & \frac{1}{3} & \frac{1}{3} \\
\frac{1}{3} & \frac{1}{3} & \frac{1}{3} \\
\frac{1}{3} & \frac{1}{3} & \frac{1}{3}
\end{array}\right)
$$

In terms of changes the gain $k_{L}$, it has been tested that when increasing $k_{L}$ from 0 to 0.01 , the overshoot, the setting time, and oscillations of the system are decreased gradually. After tuning the gain $k_{L}$ repeatedly, $k_{L}=0.016$ is finally determined to achieve a desirable system dynamics.

\section{A. Voltage Dip}

This case illustrates the voltage dip problem under heavy load conditions and the utilization of ESs to boost the voltage. When the bus 6 voltage decline to 0.94p.u., the three ESs are activated at $2 s$ by the switches. The conventional droop control is also implemented to compare with the control strategy proposed. The parameters for the conventional droop control are $m_{p}=0.016$ and $n_{q}=0.021$ respectively. For the distributed controller, the voltage references for ESs are calculated according to the consensus algorithm. The average voltage discovery process is shown in Fig.10. It can realize about $0.2 \mathrm{~s}$ updating for the voltage reference in this system. Due to the frequent updating for the voltage reference will incur the system stability issue, the fast response of the ES makes the value of 0.2 interval large enough for the system to follow and presents good dynamic performance in Fig.11.

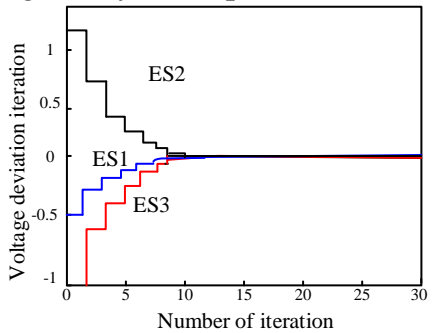

Fig. 10 Average voltage discovery

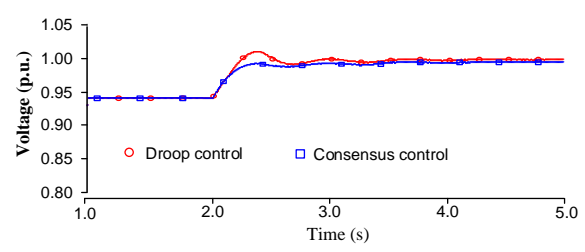

Fig. 11 Comparison for two control schemes 
TABLE 2 Comparison between the droop control and the distributed control

\begin{tabular}{|c|c|c|c|}
\hline & Voltage & $\begin{array}{c}\text { Time for steady } \\
\text { state }\end{array}$ & $\begin{array}{c}\text { Communication } \\
\text { requirements }\end{array}$ \\
\hline $\begin{array}{c}\text { Droop } \\
\text { control }\end{array}$ & 1.02 p.u. & $3.5 \mathrm{~s}$ & No \\
\hline $\begin{array}{c}\text { Consensus } \\
\text { control }\end{array}$ & $1 . p . u$. & $2.8 \mathrm{~s}$ & Sparse network \\
\hline
\end{tabular}

The conventional droop control is compared with the distributed voltage control method proposed. The droop control results in the steady-state voltage deviation without the communication. However, it will take more time for the voltage to reach the steady-state value. The droop control generates an error for voltage adjustments as well as overvoltage at the initial stage of the disturbance. In contrast, the controller proposed ensures the voltage well-regulated at 1p.u. quickly. The optimal voltage 1.0 p.u. is achieved by the consensus algorithm with the sparse communication network. Tab.2 presents the control performance of the two control strategies. It can be concluded that more accurate voltage control is achieved and the overvoltage is avoided during the transients for the improved control.

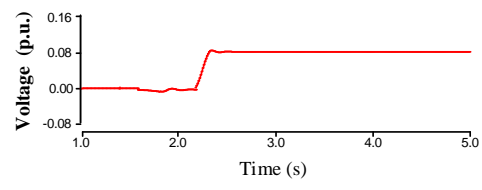

Fig. 12(a) ES voltage

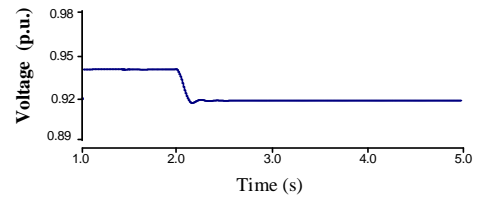

Fig. 12(b) Uncritical load voltage

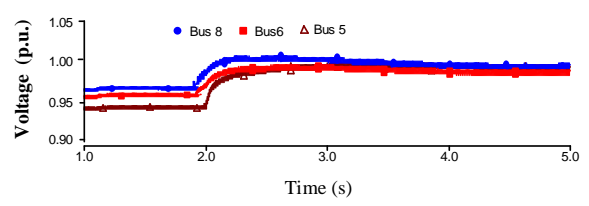

Fig. 12(c) Bus voltage

It can be observed in Fig.12 that ESs output voltage increase to 0.08p.u.in Fig.12(b) to restore the bus voltage to the voltage reference in Fig.12(c). An increase in ES voltage will result in a decrease in the uncritical load voltage from 0.94 p.u. to 0.92 p.u. shown in Fig.12(b), so as to maintain the voltage of the smart load at the rated value. The voltage support mode of ESs has been proved. In voltage support mode with an RL type NC load, the total reactive power of the smart load is equal to the difference between the reactive power produced by the ES and that consumed by the load which reduces the voltage regulation capability compared to the case of a purely resistive load.

\section{B. Wind Power Variation}

When the output power of the wind turbine G1 fluctuates from $8 \mathrm{MW}$ to $9 \mathrm{MW}$ at $2 \mathrm{~s}$ for the sake of increasing wind speed, the bus 5, 6 and 8 voltage rise without the ESs as shown in Fig.13. With the ESs activated at 3s using the control strategy proposed, the reactive power output of ESs increases quickly. The rising voltage profile of the buses are brought back to the average voltage for the sake of the reactive power consumption by ES in Fig.14. All ESs absorb the reactive power for the network resulting from the updating voltage reference. It is shown that the distributed control is effective in suppressing the voltage and can quickly bring the voltage back to the reference value given.

The active power of the smart load follows the wind power variation in Fig.15, which can be regarded as the load demand management offering a cost-effective method to match power supply and demand profiles. For an RL type NC load, better voltage regulation could be achieved in voltage suppress mode as both ES (working in inductive mode) and the NC load consume inductive reactive power and thereby, aid each other.

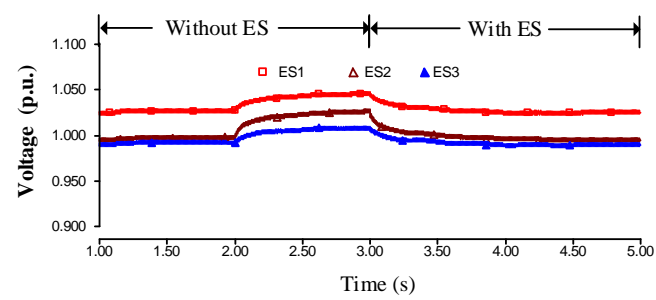

Fig. 13 Bus voltage with and without ESs

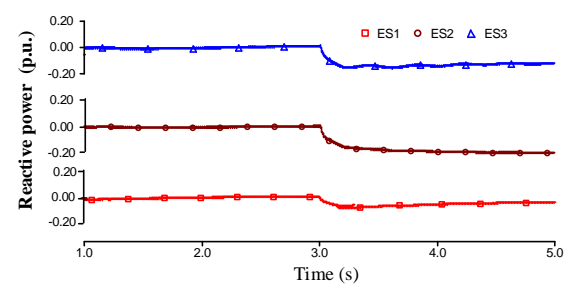

Fig. 14 Reactive power output of ESs

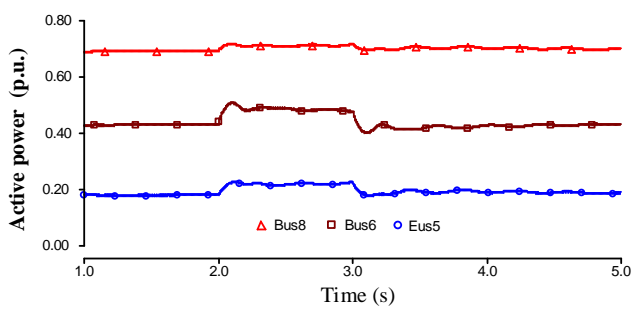

Fig. 15 Active power output of smart load

\section{Three-Phase Short-Circuit Fault}

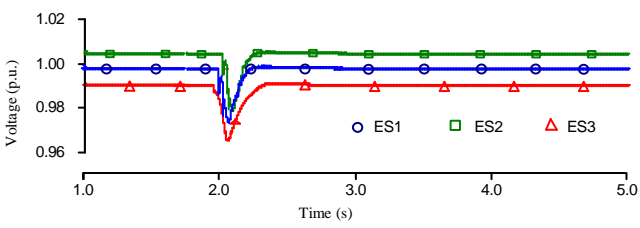

Fig. 16 Bus voltage with ESs 
A 100ms three-phase short-circuit fault is applied at the bus 5 . The voltage sag at the bus of the remaining ESs is incurred. The dynamic response of the ES is shown in Fig.16 and Fig.17. The reactive power output of ES increases to compensate the voltage dip. The bus voltage responds quickly to maintain the critical voltage level.

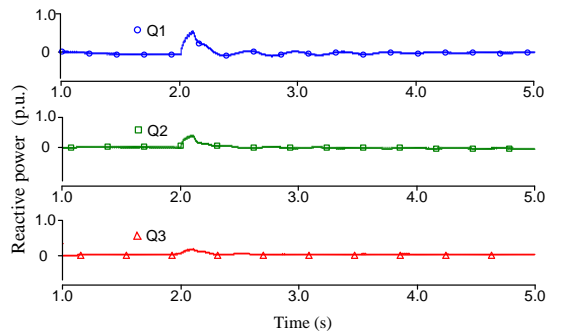

Fig. 17 Reactive power output of ESs

The consensus algorithm can ensure the voltage reference updating constantly, so does the reactive power output. It can be seen that the ESs operate fairly together to help provide dynamic reactive power support, and keep voltage profile within the acceptable range even in the extreme condition.

\section{CONCLUSIONS}

This paper proposes a novel distributed control method for adjusting the whole voltage level of ESs installed in the microgrid. To compensate the inaccuracies caused by the conventional droop control and reduce the communication cost of the central control, the distributed control strategy designed updates voltage reference for controller to realize the precise voltage control and achieve a good dynamic response using the sparse communication network. It is preferable from the technological point of view, as it avoids a few issues that would arise in the traditional control strategy. The simulation results indicate that the control strategy can coordinate the reactive power dispatch among ESs to provide the necessary voltage support during a contingency. However, a tradeoff exists between maintaining a low communication cost and achieving a high performance in reaching a consensus. It is true that the algorithm may require a long time to converge if applied to a larger power system. Further work can be focused on the algorithm improvement to accelerate the information discovery process.

\section{REFERENCES}

[1] N. Pogaku, M. Prodanovic, and T. C. Green, "Modeling, analysis and testing of autonomous operation of an inverter-based microgrid," IEEE Trans. Power Electron., vol. 22, no. 2, pp. 613-625, Mar. 2007.

[2] J. A. P. Lopes, C. L. Moreira, and A. G. Madureira, "Defining control strategies for microgrids islanded operation,” IEEE Trans. Power Syst., vol. 21, no. 2, pp. 916-924, May 2006.

[3] Mehrdad Yazdanian, and Ali Mehrizi-Sani, "Distributed Control Techniques in Microgrids,” IEEE Trans. Smart Grid, vol. 5, no. 6, pp. 916-924, Nov. 2014.

[4] S. Y. R. Hui, C. K. Lee, and F. F. Wu, "Electric springs-a new smart grid technology,” IEEE Trans. Smart Grid, vol. 3, no. 3, pp. 15521561, Sept. 2012.

[5] N. R. Chaudhuri, C.K. Lee, B. Chaudhuri, and S. Y. Ron Hui, "Dynamic Modeling of Electric Springs,” IEEE Trans. Smart Grid, vol. 5, no. 5, pp.2450-2458, Sept. 2014.

[6] S. C. Tan, C. K. Lee, and S. Y. R. Hui, "General steady-state analysis and control principle of electric springs with active and reactive power compensations,” IEEE Trans. Power Electron., vol. 28, no. 8, pp.39583969, Aug. 2013.

[7] Luo X., Akhter Z., Lee C.K., Chaudhuri B., Tan S.C. and Hui S.Y.R. "Distributed voltage control with electric springs: comparison with STATCOM”, IEEE Transactions on Smart Grid. vol. 6, no. 1, pp. 209-219, Jan. 2015.

[8] C.K. Lee and S.Y.R. Hui, "Reduction of energy storage requirements for smart grid using electric springs,” IEEE Trans. Smart Grid. vol. 4, no. 3, pp.1282-1288, Apr., 2013.

[9] X. Chen, Y. Hou, S. Tan, C. Lee, and S.Y. R. Hui, "Mitigating Voltage and Frequency Fluctuation in Microgrids Using Electric Springs," IEEE Trans. Smart Grid. vol. 6, no. 2, pp.508-515, Mar., 2015.

[10] S. Yan, S. Tan, C. K. Lee, B. Chaudhuri, and S. Y. Ron Hui, "Electric Springs for Reducing Power Imbalance in Three-Phase Power Systems," IEEE Trans. Power Electron.,vol.30, no. 7, pp. 3601-3609, July. 2015.

[11] C. K. Lee, N. R. Chaudhuri, B. Chaudhuri, and S. Y. R. Hui, "Droop control of distributed electric springs for stabilizing future power grid,” IEEE Trans. Smart Grid. vol. 4, no. 3, pp.1558-1566, Sept., 2013.

[12] Y. Mohamed and E. F. El-Saadany, "Adaptive decentralized droop controller to preserve power sharing stability of paralleled inverters in distributed generation microgrids,” IEEE Trans. Power Electron., vol. 23, no. 6, pp. 2806-2816, 2008

[13] J. M. Guerrero, J. C. Vásquez, J. Matas, M. Castilla, L. G. d. Vicuña, and M. Castilla, "Hierarchical control of droop-controlled AC and DC microgrids-A general approach toward standardization,” IEEE Trans. Ind. Electron., vol. 58, pp. 158-172, Jan. 2011.

[14] A. Bidram and A. Davoudi, "Hierarchical structure of microgrids control system,” IEEE Trans. Smart Grid, vol. 3, pp. 1963-1976, Dec. 2012.

[15] K. De Brabandere, B. Bolsens, J. Van Den Keybus, A. Woyte, J. Driesen, and R. Belmans, "A voltage and frequency droop control method for parallel inverters,” IEEE Trans. Power Electron., vol. 22, no. 4, pp. 1107-1115, Jul. 2007.

[16] Q. Zhong, "Robust droop controller for accurate proportional load haring among inverters operated in parallel,” IEEE Trans. Ind. Electron., vol. 58, 2011.

[17] W. Yao, M. Chen, J. Matas, J. Guerrero, and Z.-M. Qian, "Design and analysis of the droop control method for parallel inverters considering the impact of the complex impedance on the power sharing," IEEE Trans. Ind. Electron., vol. 58, no. 2, pp. 576-588, Feb. 2011.

[18] Lazzarin T B, Bauer G A T, Barbi I, "A control strategy for parallel operation of single-phase voltage source inverters: analysis, design and experimental results[J]. IEEE Trans. Ind. Electron., vol. 60, no. 6, pp. 2194-2204, Feb. 2013.

[19] C. C. Hang, K. J. Astrom, and W. K. Ho, "Refinements of the ZieglerNichols tuning formula," in Proc. Inst. Elect. Eng., Control Theory Appl., Mar. 1991, vol. 138, pp. 111-118, 2.

[20] R.Olfati-Saber and R.M.Murray, "Consensus problems in networks of agents with switching topology and time-delays,” IEEE Trans. Autom. Control, vol. 49, no. 9, pp. 1520-1533, 2004.

[21] V. Nasirian, S. Moayedi, A. Davoudi, and Frank L. Lewis, "Distributed Cooperative Control of DC Microgrids ," IEEE Trans. Power Electron.,vol.30, no.4, pp. 2288-2303, Apr.. 2015

[22] H. Kanchev, D. Lu, F. Colas, V. Lazarov, and B. Francois, "Energy management and operational planning of a microgrid with a PV-based active generator for smart grid applications,” IEEE Trans. Ind. Electron., vol. 58, no. 10, pp. 4583-4592, Oct. 2011.

[23] A. Maknouninejad, Z. Qu, J. Enslin, and N. Kutkut, "Clustering and cooperative control of distributed generators for maintaining microgrid unified voltage profile and complex power control," in Proc. IEEE PES Transmission and Distribution Conference and Exposition , Orlando, FL, 2012, pp. 1-8.

[24] Z. Qu, J. Wang, and R. Hull, "Cooperative control of dynamical systems with application to autonomous vehicles," IEEE Trans. Autom. Control, vol. 53, no. 4, pp. 894-911, May 2008.

[25] A. Bidram, A. Davoudi, F. L. Lewis, and J. M. Guerrero, "Distributed cooperative control of microgrids using feedback linearization," IEEE Trans. Power Syst., vol. 28, pp. 3462-3470, Aug. 2013.

[26] A. Bidram, A. Davoudi, F.L. Lewis, Qu. Zhihua, "Secondary control of microgrids based on distributed cooperative control of multi-agent systems”, IET Gener. Transm. Distrib., vol.7, no.8, pp.822-831, 2013. 
[27] R. Olfati-Saber, J. Fax, and R. M. Murray, "Consensus and cooperation in networked multi-agent systems," Proceedings of the IEEE, vol. 95, pp. 215-233, Jan. 2007. 